Pacific Journal of Mathematic 


\title{
ON THE NUMBER OF NON-ALMOST ISOMORPHIC MODELS OF $T$ IN A POWER
}

\author{
SAHARON SHELAH
}

Let $T$ be a first order theory. Two models are almost isomorphic if they are elementarily equivalent in the language $L_{\infty, \omega}$. We investigate the number of non almost-isomorphic models of $T$ of power $\lambda$ as a function of $\lambda, I(T, \lambda)$. We prove $\mu>\lambda \geqq|T|, I(T, \lambda) \leqq \lambda$ implies $I(T, \mu) \leqq I(T, \lambda)$. In fact, we generalize the downward Skolem-Lowenheim theorem for infinitary languages. Th. $(1,4,5)$.

Let $L$ be a set of predicates with finite number of places and sufficient number of variables. (We assume there are no function symbols in $L$ for simplicity only.) $|L|$ will denote the number of predicates in $L$ plus $\boldsymbol{\aleph}_{0}$. Models will be denoted by $M, N$. The set of elements of $M$ will be $|M|$, the cardinality of a set $A$ by $|A|$ and so the cardinality of $M$ by $\|M\|$. Unless specified otherwise, every model is an $L$-model. Cardinals will be denoted by $\lambda, \mu, \kappa, \chi$ ordinals $i, j, \alpha, \beta$. $T$ will denote a theory, i.e., set of sentences. We define $\mu^{(\lambda)}=\sum_{\kappa<\lambda} \mu^{\kappa}$. For cardinals $\lambda, \mu$ we define the language $L(\lambda, \mu)$ i.e., a set of formulas. This set is defined as the well known first-order language where we adjoin to its operations conjunction and disjunction on a set of $<\lambda$ formulas (i.e., $\bigwedge_{i \in I} \phi_{i}$, where $|I|<\lambda$ ) and existential or universal quantifications over a sequence of $<\mu$ variables. $L^{*}(\lambda, \mu)$ will be defined as $L(\lambda, \mu)$ where in addition we permit quantification of the form

$$
\left.\left[\forall \bar{x}^{1}\right)\left(\exists \bar{y}^{1}\right) \cdots\left(\forall \bar{x}^{n}\right)\left(\exists \bar{y}^{n}\right) \cdots\right]_{n<\omega}
$$

if

$$
\left|\left\{x_{0}^{1}, x_{1}^{1}, \cdots, y_{0}^{1}, y_{1}^{1}, \cdots, x_{0}^{n} \cdots\right\}\right|<\mu .
$$

$R L^{*}(\lambda, \mu)$ will denote the subset of $L^{*}(\lambda, \mu)$ consisting of the formulas $\Phi$ of $L^{*}(\lambda, \mu)$ such that for every subformula $\phi$ of $\Phi$, if $\phi=\left[\left(\forall \bar{x}^{1}\right)\right.$ $\left.\left(\exists \bar{y}^{1}\right) \cdots\right] \psi$, then $\vDash \phi \leftrightarrow>\left[\left(\exists \bar{x}^{1}\right)\left(\forall \bar{y}^{1}\right) \cdots\right]>\psi$. Clearly $R L^{*}(\lambda, \mu) \supset$ $L(\lambda, \mu) . \quad K$ will denote any of those languages. Satisfaction (i.e., if $\dot{\phi}=\dot{\phi}(\bar{x})$, and $\bar{a}$ is a sequence from $|M|$, then $M \vDash \phi[\bar{a}])$ is defined naturally. (See Hanf [2] and Henkin [3].) The only nontotally trivial case is

$$
\psi(\bar{z})=\left[\left(\forall \bar{x}^{0}\right)\left(\exists \bar{y}^{0}\right)\left(\forall \bar{x}^{1}\right)\left(\exists \bar{y}^{1}\right) \cdots\right] \phi\left(\bar{z}, \bar{x}^{0}, \bar{x}^{1}, \cdots, \bar{y}^{0}, \bar{y}^{1} \cdots\right) .
$$

$M \vDash \psi[\bar{a}]$ if and only if there are functions $f_{i}^{n}\left(\bar{x}^{0}, \cdots, \bar{x}^{n}\right)$ such that for every sequence $\bar{a}^{0}, \bar{a}^{1}, \ldots$ from $M, M \vDash \phi\left[\bar{a}, \bar{a}^{0}, \bar{a}^{2}, \cdots, \bar{b}^{0}, \bar{b}^{1}, \cdots\right]$ where $\bar{b}^{n}=\left\langle\cdots, f_{i}^{n}\left(\bar{a}^{0}, \bar{a}^{1}, \cdots, \bar{a}^{n}\right), \cdots\right\rangle$. For a sentence $\psi^{\prime}, \vDash \psi$ if for 
every $M, M \vDash \psi$. (Such languages were first defined in Henkin [3].)

If $\Gamma$ is a set of formulas (for example one of the languages defined above), $M$ is a $\Gamma$ elementary submodel of $N$, if the set of elements of $M,|M|$ is included in the set of elements of $N,|N|$, and for every formula $\phi(\bar{x}), \phi(\bar{x}) \in \Gamma$, and sequence $\bar{a}$ from $|M|, M \vDash \phi[\bar{a}]$ if and only if $N \vDash \phi[\bar{a}], M, N$ are $\Gamma$-elementarily equivalent if for every sentence $\phi \in \Gamma, M \vDash \phi$ if and only if $N \vDash \phi$.

THeorem 1. Let $\lambda>\mu, \lambda$ regular and $T$ be a theory in $R L^{*}(\lambda, \mu)$ [i.e., $T \subset R L^{*}(\lambda, \mu)$ ] and $\Gamma$ be the set of subformulas of the formulas in $T$. Then for every model $M$ we can add $<\lambda+|T|^{+}$functions of $<\mu$ places such that: If $A \subset M$, and $A$ is closed under those functions, then there exists a $\Gamma$-elementary submodel $N$ of $M,|N|=A$. So if $\kappa \geqq \lambda+|T|$ (or $\kappa \geqq$ the number of those functions) and $\kappa^{(\mu)}=\kappa$, and $T$ has a model of power $\geqq \kappa$, then $T$ has a model of power $\kappa$.

Proof. This theorem is proved in [9], and is a straight-forward generalization of a theorem of Hanf in [2].

DEFINITION 1.

$$
\begin{aligned}
L(\infty, \mu) & =\bigcup_{\lambda} L(\lambda, \mu), L^{*}(\infty, \mu)=\bigcup_{\lambda} L^{*}(\lambda, \mu), \\
R L^{*}(\infty, \mu) & =\bigcup_{\lambda} R L^{*}(\lambda, \mu) .
\end{aligned}
$$

Definition 2. (1) $M$ and $N$ are $\mu$-almost isomorphic, $M \sim_{\mu} N$ if $M, N$ are $L(\infty, \mu)$-elementarily equivalent. We say $M$ and $N$ are almost isomorphic if $M \sim_{\aleph_{0}} N$, and we write $M \sim N$.

(2) $I(T, \lambda, \mu)$, is the number of non- $\mu$-almost-isomorphic models of $T$ of power $\lambda$. We assume always $\lambda$ is $\geqq$ then $|T|$.

See footnote 1.

TheOREm 2. If $T$ is a theory in $R L^{*}(\lambda, \mu), \mu=\boldsymbol{\aleph}_{0}$ or $\mu=\mu_{1}^{+}$, $\kappa \geq \chi=\chi^{(\mu)}+\lambda+|T|$ and $I(T, \chi, \mu) \leqq \chi$ then $I(T, \kappa, \mu) \leqq I(T, \chi, \mu)$.

The proof is broken into a series of lemmas.

Remarks. (1) It is not hard to show that if $T \subset L\left(\lambda, \boldsymbol{\aleph}_{0}\right)$, $I\left(T, \chi, \boldsymbol{\aleph}_{0}\right) \leqq \chi$, then for every $\kappa_{1}, \kappa_{2} \geqq \beth_{\left(2^{\lambda+\chi}\right)+}, I\left(T, \kappa_{1}, \boldsymbol{\aleph}_{0}\right)=I\left(T, \kappa_{2}\right.$, $\boldsymbol{N}_{0}$ ). (See Makkai [7] and Eklof [15].)

1 The results here appear in the notices [10] Th. 5 [11] Th. 3. The lemma has other uses: see [12] Th. 2.5 and Remark (4): in [11] their consequences are better formulated. In Th. 2 we can replace $T \subset R T^{*}(\lambda, \mu)$ by $T \subset R L^{*}\left(\lambda^{+}, \mu\right)$ and similarly in other cases. 
(2) Let $\lambda=\mu=\boldsymbol{\aleph}_{0}$ and suppose $|T| \leqq \kappa_{0}$. Then as the class of such theories is a set, there is a number $\kappa=H A I_{\kappa_{0}}$ (Hanf number of almost isomorphism) such that: for all $T,|T| \leqq \kappa_{0}, I\left(T, \kappa, \boldsymbol{\aleph}_{0}\right) \leqq \kappa$ if and only if there is a $\chi, I\left(T, \chi, \boldsymbol{\aleph}_{0}\right) \leqq \chi$, and $\kappa$ is the first such cardinality. (The existence of such numbers for a wide class of cases was proved by Hanf in [2].)

Question 1. What is $H A I_{\kappa_{0}}$ ? (Clearly if $\lambda \rightarrow\left(\kappa_{0}^{+}\right)_{2^{\kappa_{0}}}^{<\omega}$ then $H A I_{\kappa_{0}}<\lambda$ ).

(3) It is known that $M \sim N, \boldsymbol{\aleph}_{0}=\|M\|=\|N\|$ implies that $M$, $N$ are isomorphic (see Scott [8]).

(4) Ehrenfeucht in [1] defined a model to be rigid if it has no nontrivial automorphisms and tried to investigate what can be the class of cardinals in which a certain theory has a rigid model. $\mathrm{He}$ gives some examples, but does not prove any theorem of the form: If $T$ has a rigid model of one power, then it has a rigid model in another power.

Definition. $M$ is $\mu$-rigid if there do not exist two different sequences of length $\left\langle\mu, \bar{a}, \bar{b}\right.$, such that $(M, \bar{a}) \sim_{\mu}(M, \bar{b}) . \quad((M, \bar{a})$ is the model $M$ when we adjoin the $a$ 's as individual constants.) See footnote 2. Clearly

TheOREM. If $\mu<\lambda$, and $M$ is $\mu$-rigid, then it is $\lambda$-rigid and also rigid. By a proof similar to that of Theorem 2, we can prove:

THEOREM. If a first-order theory $T$ has a $\mu$-rigid model of power $\lambda,|T|+\boldsymbol{\aleph}_{0} \leqq \kappa=\kappa^{(\mu)} \leqq \lambda, \mu=\mu_{1}^{+}$or $\mu=\boldsymbol{\aleph}_{0}$, then $T$ has a $\mu$-rigid model of power $\kappa$.

\section{Proof of Theorem 2.}

Definition 3. (1) Let $L_{1}$ be $L$ where we adjoin to it one twoplace predicate $E$ and variables $y, y_{0}, y_{1}, \cdots$ we assume $E, y, y_{0} \cdots \neq L$. We shall write $x E y$ instead $E(x, y)$.

(2) If $R \in L$ then $R^{M}$ will denote the relation of $M$ corresponding to $R$.

(3) Let $\left\{M_{i}: i \in I\right\}$ be a set of $L$-models and we define their sum $N=\bigoplus_{i \in I} M_{i}$, (or $\oplus\left\{M_{i}: i \in I\right\}$ ). For simplicity we assume that the sets $\left|M_{i}\right|$ are pairwise disjoint. $N$ will be an $L_{1}$-model $|N|=\bigcup_{i \in I}\left|M_{i}\right|$, $R^{N}=\bigcup_{i \in I} R^{M i}$ for every $R \in L$, and $E^{N}=\left\{\langle a, b\rangle:(\exists i)\left[a, b \in\left|M_{i}\right|\right]\right\}$.

(4) For every formula $\phi$ of any language, we define by induction

${ }^{2}$ Barwise [14] suggests a similar definition and argues its naturality. 
$\bar{\phi}$ : if $\phi$ is atomic $\bar{\phi}=\phi ; \overline{\gamma \phi}=7 \bar{\phi}, \overline{\phi \bigvee \psi}=\bar{\phi} \mathrm{V} \bar{\psi}$, (likewise for the other connectives), $\overline{\exists(\exists \bar{x}) \phi}=(\exists \bar{x})\left[\bar{\phi} \wedge \Lambda_{i} x_{i} E y\right]$, (where $\left.\bar{x}=\left\langle\cdots x_{i} \cdots\right\rangle\right)$ $\overline{(\forall \bar{x}) \dot{\phi}}=(\forall \bar{x})\left[\Lambda_{i} x_{i} E y \rightarrow \bar{\phi}\right]$, and

$$
\overline{\left[\left(\forall \bar{x}^{1}\right)\left(\exists \bar{y}^{1}\right) \cdots\right] \phi}=\left[\left(\forall \bar{x}^{1}\right)\left(\exists \bar{y}^{1}\right) \cdots\right]\left(\bigwedge_{i, n} x_{i}^{n} E y \rightarrow \bar{\phi} \wedge \bigwedge_{i, n} y_{i}^{n} E y\right)
$$

if the language contains such formulas. Clearly for any language $K, \phi \in K \Rightarrow \bar{\phi} \in K$. Also, if $\phi$ is a sentence $(\forall y) \bar{\phi}$ is a sentence.

(5) Define

$\bar{T}=\{(\forall y) \bar{\phi}: \phi \in T\} \cup\left\{(\forall x) x E x,\left(\forall x_{0} x_{1} x_{2}\right)\left(x_{0} E x_{1} \wedge x_{0} E x_{2} \rightarrow x_{1} E x_{2}\right)\right\}$.

Lemma 3. Each $M_{i}$ is an L-model of $T$ if and only if $\bigoplus_{i \in I} M_{i}$ is an $L_{1}$-model of $\bar{T}$.

Proof. Immediate

DeFinition 4.

$$
\begin{aligned}
& \psi_{\alpha}^{n}=\psi_{\alpha}^{n}\left(\bar{x}^{0}, \bar{x}^{1}, \cdots, \bar{x}^{n}, \bar{y}^{0}, \cdots, \bar{y}^{n}\right)=\bigwedge\left\{R\left(x_{j_{1}}^{i_{1}}, \cdots, x_{j_{k}}^{i_{k}} \cdots\right)\right. \\
& \quad \leftrightarrow R\left(y_{j_{1}}^{i_{1}}, \cdots, y_{j_{k}}^{i_{k}} \cdots\right): i_{1}, \cdots, i_{k} \cdots \in\{0, \cdots, n\}, \\
& \left.\quad R \in L, j_{1}, \cdots, j_{k} \cdots<\alpha\right\}
\end{aligned}
$$

where

$$
\bar{x}^{n}=\left\langle\cdots x_{i}^{n} \cdots\right\rangle_{i<\alpha}, \bar{y}^{n}=\left\langle\cdots y_{i}^{n} \cdots\right\rangle_{i<\alpha} .
$$

Also let

$$
\begin{aligned}
\Phi_{\alpha}^{m}=\left[\bigwedge_{\substack{i<\alpha \\
2 n<m}} x_{i}^{2 n} E x\right. & \left.\wedge \bigwedge_{\substack{i<\alpha \\
2 n+1<m}} y_{i}^{2 n+1} E y\right] \rightarrow\left[\bigwedge_{\substack{i<\alpha \\
2 n+1<m}} x_{i}^{2 n+1} E x \wedge \bigwedge_{\substack{i<\alpha \\
2 n<m}} y_{i}^{2 n} E y\right. \\
& \left.\wedge \bigwedge_{n<m} \psi_{\alpha}^{n}\left(\bar{x}^{0}, \cdots, \bar{x}^{n}, \bar{y}^{0}, \cdots, \bar{y}^{n}\right)\right]: \\
\phi_{\alpha}^{\omega}= & \bigwedge_{m<\omega} \Phi_{\alpha}^{m}=\phi_{\alpha}^{\omega}\left(x, y, \bar{x}^{0}, \bar{y}^{0}, \bar{x}^{1}, \bar{y}^{1}, \cdots\right) .
\end{aligned}
$$

For even $n$

$$
\phi_{\alpha}^{n}=\phi_{\alpha}^{n}\left(x, y, \bar{x}^{0}, \bar{y}^{0}, \cdots, \bar{x}^{n-1}, \bar{y}^{n-1}\right)=\left[\left(\forall \bar{x}^{n}\right)\left(\exists \bar{y}^{n}\right)\left(\forall \bar{y}^{n+1}\right)\left(\exists \bar{y}^{n+1}\right) \cdots\right] \phi_{\alpha}^{\omega} .
$$

For odd $n$

$$
\phi_{\alpha}^{n}\left(x, y, \bar{x}^{0}, \bar{y}^{0}, \cdots, \bar{x}^{n-1}, \bar{y}^{n-1}\right)=\left[\left(\forall \bar{y}^{n}\right)\left(\exists \bar{x}^{n}\right)\left(\forall \bar{x}^{n+1}\right)\left(\exists \bar{y}^{n+1}\right)\left(\forall \bar{y}^{n+2}\right) \cdots\right] \phi_{\alpha}^{\omega} .
$$

\section{LEMMA 4. If}

$$
a \in|M|, b \in|N|, M, N \in\left\{M_{i}: i \in I\right\}, M^{*}=\bigoplus_{i \in I} M_{i},
$$

and $\mu=\kappa^{+}$or $\mu=\boldsymbol{\aleph}_{0}$, and $\kappa$ is finite, then $M \sim_{\mu} N$ if and only if $M^{*} \vDash \phi_{\kappa}^{0}[a, b]$. 
REMARK. Keisler in [5] used sentences similar to $\phi_{\alpha}^{n}$. These sentences can be seen as asserting something about an appropriate game (between a player choosing $\bar{x}^{0}, y^{1}, x^{2}, \cdots$ and a player choosing $\bar{y}^{0}$, $\left.\bar{x}^{1}, \cdots\right)$. In this presentation a similar theorem appears in Karp [4].

Added in proof. See also Benda [13].

Proof.

Part $A$ - Suppose $M \sim{ }_{\mu} N$.

For every two sequences $\bar{a}, \bar{b}$ of elements of $M$, either there is a formula $\phi_{\bar{a}, \bar{b}}(\bar{x})$ of $L(\infty, \mu)$ such that $M \vDash \dot{\phi}_{\bar{a}, \bar{b}}[\bar{a}], M \vDash>\dot{\phi}_{\bar{a}, \bar{b}}[\bar{b}]$, or there is no such $\phi$ and in this case, we let $\phi_{\bar{a}, \bar{b}}(\bar{x})=\left(x_{0}=x_{0}\right)$.

Let $\dot{\phi}_{\bar{a}}^{\prime}(\bar{x})=\Lambda_{\bar{b}} \dot{\phi}_{\bar{a}, \bar{b}}(\bar{x}) \in L(\infty, \mu)$. Let $\overline{\phi_{\bar{a}}^{-}(\bar{x})}=\dot{\phi}_{\bar{a}}^{\prime}(y, \bar{x})$. Let $\alpha<\mu$. We define the functions

$$
\begin{aligned}
& f_{i}^{2 n}\left(\bar{x}^{0}, \bar{y}^{0}, \bar{y}^{1}, \bar{x}^{1}, \bar{x}^{2}, \cdots, \bar{y}^{2 n-1}, \bar{x}^{2 n-1}, \bar{x}^{2 n}\right), \\
& f_{i}^{2 n+1}\left(\bar{x}^{0}, \bar{y}^{0}, \bar{y}^{1}, \bar{x}^{1}, \bar{x}^{2}, \cdots, \bar{x}^{2 n}, \bar{y}^{2 n}, \bar{y}^{2 n+1}\right)
\end{aligned}
$$

for $i<\alpha$ such that: If $\bar{a}^{0}, \bar{b}^{0}, \bar{a}^{1}, \bar{b}^{1} \cdots$ are sequences of length $\alpha, \bar{a}^{2 n}$ a sequence of elements of $M$, and $\bar{b}^{2 n+1}$ a sequence of elements of $N$, and for every $n$

$$
\begin{aligned}
\bar{b}^{2 n} & =\left\langle\cdots f_{i}^{2 n}\left(\bar{a}^{0}, \bar{b}^{0}, \cdots, \bar{a}^{2 n}\right) \cdots\right\rangle_{i<\alpha} \\
\bar{a}^{2 n+1} & =\left\langle\cdots f_{i}^{2 n+1}\left(\bar{a}^{0}, \cdots, \bar{b}^{2 n+1}\right) \cdots\right\rangle_{i<\alpha}
\end{aligned}
$$

then $M^{*} \vDash \dot{\phi}_{a}^{\omega}\left[a, b, \bar{a}^{0}, \bar{b}^{0}, \cdots\right]$.

Suppose we have defined $f_{\imath}^{n}$ for $n<2 m$, and let us define $f_{2}^{2 m}$ for $i<\alpha . \quad\left(f_{i}^{2 m+1}\right.$ are defined similarly. $)$

If for some $n<2 m, i<\alpha b_{\imath}^{n} \notin|N|$, or for some $i<\alpha, n \leqq 2 m a_{i}^{n} \notin$ $|M|$, then $f_{i}^{2 m}\left(\bar{\alpha}^{0}, \cdots, a^{2 m}\right)$ is defined as an arbitrary element of $M^{*}$. Also if there exists a formula $\psi\left(\bar{z}^{1}, \cdots, \bar{z}^{n}\right) \in L(\infty, \mu)$ such that

$$
M \vDash \psi\left[\bar{a}^{0}, \bar{a}^{1}, \cdots, \bar{a}^{2 m-1}\right] N \vDash>\psi\left[\bar{b}^{0}, \cdots, \bar{b}^{2 m-1}\right],
$$

we define $f_{i}^{2 m}\left(\bar{\alpha}^{0} f^{0} \cdots \bar{\alpha}^{2 m}\right)$ arbitrarily.

So assume none of the previous cases occur. Define $\bar{a}[n]=\bar{a}^{0} \frown$ $\bar{a}^{1} \frown \cdots \frown \bar{a}^{n}$ (the concatenation of $\bar{a}_{1}, \cdots, \bar{a}^{n}$ ) and $\bar{b}[n]=\bar{b}^{0} \frown \cdots \frown \bar{b}^{n}$. Clearly

$$
M \vDash(\forall \bar{x})\left(\phi_{\bar{a}[2 m-1]}(\bar{x}) \rightarrow(\exists \bar{z}) \phi_{\bar{a}[2 m]}(\bar{x}, \bar{z})\right) .
$$

As $M \sim{ }_{\mu} N, N$ also satisfies the above sentence; so there exists $\bar{b}^{2 m}$ such that for every $\phi \in L(\infty, \mu), M \vDash \phi\left[\bar{a}^{0}, \cdots, \bar{a}^{2 m}\right]$ if and only if $N \vDash \phi\left[\bar{b}^{0}, \cdots, \bar{\phi}^{2 m}\right]$. Let $f_{i}^{2 m}\left(\bar{a}^{0}, \bar{b}^{0}, \cdots, \bar{a}^{2 m}\right)=\bar{b}_{i}^{2 m}$. 
Clearly this shows that $M^{*} \vDash \phi_{\alpha}^{0}[a, b]$ for every $\alpha<\mu$, and in particular for $\kappa$.

Part $B$. We now assume that $M^{*} \vDash \phi_{1}^{0}[a, b]$, and $\mu=\aleph_{0}$. The proof in the case $\mu=\kappa^{+}$or $1<\kappa<\boldsymbol{\aleph}_{0}$ is similar. For simplicity, we shall not distinguish between $\bar{a}=\left\langle a_{0}\right\rangle$ and $a_{0}$.

Two sequences, $\bar{a}$ from $M$ and $\bar{b}$ from $N$, of length $n, n<\omega$, will be called equivalent if $M^{*} \vDash \phi_{1}^{n}[a, b, \bar{a}, \bar{b}]$. If $n=2 m$, clearly for every $b^{n+1} \in|N|$ there exists $a^{n+1} \in|M|$ such that $\bar{a} \frown\left\langle a^{n+1}\right\rangle$ and $\bar{b} \frown$ $\left\langle b^{n+1}\right\rangle$ are equivalent, and similarly for $n=2 m+1$.

Let $\phi(\bar{x}) \in L(\infty, \mu), \bar{x}$ a finite sequence of variables. We shall prove that if $\bar{a}, \bar{b}$ are equivalent then $M \vDash \phi[\bar{a}]$ if and only if $N \vDash \phi[\bar{b}]$. As subformulas of formulas with $<\boldsymbol{\aleph}_{0}$ free variables have $<\boldsymbol{\aleph}_{0}$ free variables we can prove it by induction. For atomic formulas it follows from the definition of $\phi_{1}^{n}$. For $7 \phi, \phi \vee \psi$, it is immediate, and so also for the other connectives. For quantification it follows by the fact mentioned above after the definition of equivalent sequences.

So we have proved that if $\bar{a}, \bar{b}$ are equivalent sequences, $\phi(\bar{x}) \epsilon$ $L(\infty, \mu)$, then $M \vDash \phi[\bar{\alpha}]$ if and only if $N \vDash \phi[\bar{b}]$. Since the sequences of length zero from $M$ and $N$ are equivalent (by our hypotheses $M^{*} \vDash$ $\left.\phi_{1}^{0}(a, b)\right)$, we get our conclusion that $M \sim N$. This proves Lemma 4 .

Lemma $5 . \quad \phi_{\alpha}^{0}(x, y) \in R L^{*}(\infty, \mu)$. See footnote 3 .

Proof. It is easily seen that the only thing we have to prove is:

$$
\vDash\left[\left(\forall \bar{x}^{0}\right)\left(\exists \bar{y}^{0}\right)\left(\forall y^{1}\right)\left(\exists x^{1}\right) \cdots\right] \bigwedge_{n<\omega} \phi_{\alpha}^{n} \leftrightarrow 7\left[\left(\exists \bar{x}^{0}\right)\left(\forall \bar{y}^{0}\right)\left(\exists \bar{y}^{1}\right)\left(\forall x^{1}\right) \cdots\right] \underset{n<\omega}{\bigvee}>\phi_{\alpha}^{n}
$$

For simplicity, let $\alpha=1$.

It is not hard to see that if $M \models\left[\left(\forall x^{0}\right)\left(\exists y^{0}\right) \cdots\right] \Lambda_{n<\omega} \phi_{1}^{n}$, then $M \vDash>\left[\left(\exists x^{0}\right)\left(\forall y^{0}\right) \cdots\right] \bigvee_{n<\omega}>\phi_{1}^{n}$. (See, for example, Keisler [6].)

So suppose $M \vDash>\left[\left(\exists \bar{x}^{0}\right)\left(\forall y^{0}\right) \cdots\right] \mathbf{V}_{n<\omega}>\phi_{1}^{n}$. It is not hard to see that for every $n<\omega$, and formula $\phi$

$$
\begin{aligned}
& \vDash>\left[\left(\forall z_{1}\right)\left(\exists z_{2}\right)\left(\forall z_{3}\right) \cdots\right] \phi \leftrightarrow\left(\exists z_{1}\right)>\left[\left(\exists z_{2}\right)\left(\forall z_{3}\right) \cdots\right] \phi \\
& \vDash\left(\exists z_{1}\right)>\left[\left(\exists z_{2}\right)\left(\forall z_{3}\right) \cdots\right] \phi \leftrightarrow\left(\exists z_{1}\right)\left(\forall z_{2}\right)>\left[\left(\forall z_{3}\right) \cdots\right] \phi,
\end{aligned}
$$

etc.

Now let us define functions $g_{n}\left(x^{0}, y^{0}, y^{1}, \cdots, x^{i} \cdots y^{j} \cdots\right)_{i, j<n}$. Let $\left.\theta_{n}\left(x, y, x^{0}, y^{0}, x^{1}, y^{1}, \cdots, x^{n}, y^{n}\right)=>\left[\forall x^{n}\right)\left(\exists y^{n}\right)\left(\forall y^{n+1}\right)\left(\exists x^{n+1}\right) \cdots\right] \underset{n<\omega}{\mathbf{V}}>\dot{\phi}_{1}^{n} \cdot$

${ }^{3}$ This lemma is, in fact, a translation of a well known theorem from game theory. 
(This is for even $n$, the definition for odd $n$ is clear.) The functions will be such that if $a^{0}, \cdots, a^{n} \in|M|, b^{0}, \cdots, b^{n} \in|N|$, and for every $2 m \leqq n b^{2 m}=g_{2 m}\left(a^{0}, b^{0}, \cdots\right)$, and for every $2 m+1 \leqq n a^{2 m+1}=g_{2 m+1}\left(a^{0}\right.$, $\left.b^{0}, \cdots\right)$; then $M^{*} \vDash \theta_{n}\left[a, b, a^{0}, b^{0} \cdots\right]$. The definition is self-evident. Let $a^{0} \cdots a^{n} \cdots \in|M|, b^{0} \cdots b^{n} \cdots \in|N|$ be such that for every $2 m b^{2 m}=g_{2 m}\left(a^{0}, b^{0} \cdots\right)$ and for every $2 m+1 a^{2 m+1}=g_{2 m+1}\left(a^{0}, b^{0} \cdots\right)$ and let $n<\omega$. As $M^{*} \vDash \theta_{n+1}\left[a, b, a^{0}, b^{0} \cdots a^{n}, b^{n}\right]$, clearly $M^{*} \vDash \phi_{1}^{n}\left(a, b, a^{0}\right.$, $\left.b^{0} \cdots a^{n}, b^{n}\right)$.

So $M^{*} \vDash \bigwedge_{n<\omega} \phi_{1}^{n}\left(a, b, a^{0}, b^{0}, \cdots, a^{n} b^{n}\right)$, and hence $M^{*} \vDash \phi_{1}^{\omega}\left[a, b, a^{0}\right.$, $\left.b^{0} \cdots\right]$. So $M^{*} \vDash \phi_{1}^{0}[a, b]$ (as this is true for every $a^{0}, b^{1}, a^{2}, b^{3} \cdots$ ) and this is the desired conclusion.

Lemma 6. Let $\mu=\kappa^{+}$or $\mu=\boldsymbol{\aleph}_{0}, \kappa=1, T$ a theory in $R L^{*}(\lambda, \mu)$, $\chi=\chi^{(\mu)}+\lambda+|T|$, and $I(T, \chi, \mu) \leqq \chi$. Then for every model $N$ of $T$ of power $>\chi$, there exists a model $M$ of $T$ of power $\chi$ such that $M \sim_{\mu} N$.

REMARK. This clearly proves Theorem 2.

Proof. Let $\left\{M_{i}: i \in I\right\}$ be a maximal set of non- $\mu$-almost-isomorphic models of $T$ of power $\chi$, and let $N$ be a model of $T$ of power $>\chi$ such that for no $i \in I, N \sim_{\mu} M_{i}$.

Let $M^{*}=\bigoplus\left(\{N\}\left\{M_{i}: i \in I\right\}\right)$. Clearly $M^{*}$ is a model of $T_{1}=\bar{T} U$ $\left\{(\forall x, y)\left[>x E y \rightarrow>\phi_{k}^{0}(x, y)\right]\right\}$. Let $a \in|N|$, and $A=\{a\} \cup \cup\left\{\left|M_{i}\right|: i \in I\right\}$. Clearly, $|A|=\chi$.

Let $\Gamma$ be the set of subformulas of formulas $\in T_{1}$. By Theorem 1, it follows that $M^{*}$ has a $\Gamma$-elementary submodel $N^{*},\left|N^{*}\right| \supset A, \chi=$ $\left\|N^{*}\right\|=$ (the power of $N^{*}$ ), such that every equivalence class (of $E$ ) in $N^{*}$ has exactly $\chi$ elements. Clearly, $N^{*}=\bigoplus\left(\left\{N_{1}\right\} \cup\left\{M_{i}: i \in I\right\}\right)$, and for every $i, N_{1}, M_{i}$ are models of $T$, and they are non- $\mu$-almost-isomorphic. So $N_{1}$ contradicts the definition of $\left\{M_{i}: i \in I\right\}$, thus proving Lemma 6 .

This ends the proof of Theorem 2 .

\section{REFERENCES}

TM will denote the Proc. 1963 Berkeley Symposium on Theory of models, North Holland Publ. Co., 1965.

1. A. Ehrenfeucht, Elementary Theories with Models Without Automorphisms, TM, pp. $70-76$.

2. W. Hanf, Doctoral Dissertation, University of California, 1962.

3. L. Henkin, Some Remarks on Infinitely Long Formulas, Infinitistic Methods, Warsaw, (1961), 167-183. 
4. C. Karp, Finite Quantifier Equivalence, TM, pp. 407-412.

5. H. J. Keisler, Some applications of infinitely long formulas, J. Symbolic Logic 30 (1965), 339-349.

6. - Formulas With Linearly Ordered Quantifiers, Lecture Notes in Math. 72. The Syntex and Semantics of Infinitary Languages, (1968), 96-131.

7. M. Makkai, Notices of A.M.S., vol. 16 (1964), p. 322.

8. D. Scott, Logic with denumerable long formulas and finite strings of quantifiers, TM, pp. 329-341.

9. S. Shelah, Master's thesis written under the guidence of Professor H. Gaifman. The Hebrew Univ. Jerusalem 1967.

10. On the number of the non-isomorphic models of a theory in a cardinality, Notices of Amer. Math. Soc., 17 (1970), 576.

11. Some unconnected results in model theory, Notices of the Amer. Math. Soc., 18 (1971), 576. April.

12. — A combinatorial problem, stability and order for models and theories in infinitary languages, to appear (Pacific J. Math.)

13. M. Benda, Reduced products and nonstandard logics, J. Symbolic Logic, 34 (1969), 424-436.

14. J. Barwise, Back and forth thru infinitary logic, in a forthcoming book edited by Morley.

15. P. C. Eklof, On the existence of $L_{\infty, k}$-indiscernibles, Proc. Amer. Math. Soc., 25 (1970), 798-800.

Received August 19, 1969.

The Hebrew University

AND

The University of California, Los Angeles 


\title{
PACIFIC JOURNAL OF MATHEMATICS
}

\section{EDITORS}

H. SAMELSON

Stanford University

Stanford, California 94305

C. R. HobвY

University of Washington

Seattle, Washington 98105

\section{J. DugunduI}

Department of Mathematics

University of Southern California

Los Angeles, California 90007

RICHARD ARENS

University of California

Los Angeles, California 90024

\section{ASSOCIATE EDITORS}
E. F. BECKENBACH
B. H. Neumann
F. WOLE
K. Yoshida

\section{SUPPORTING INSTITUTIONS}

\author{
UNIVERSITY OF BRITISH COLUMBIA \\ CALIFORNIA INSTITUTE OF TECHNOLOGY \\ UNIVERSITY OF CALIFORNIA \\ MONTANA STATE UNIVERSITY \\ UNIVERSITY OF NEVADA \\ NEW MEXICO STATE UNIVERSITY \\ OREGON STATE UNIVERSITY \\ UNIVERSITY OF OREGON \\ OSAKA UNIVERSITY \\ UNIVERSITY OF SOUTHERN CALIFORNIA
}

\author{
STANFORD UNIVERSITY \\ UNIVERSITY OF TOKYO \\ UNIVERSITY OF UTAH \\ WASHINGTON STATE UNIVERSITY \\ UNIVERSITY OF WASHINGTON

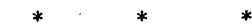 \\ AMERICAN MATHEMATICAL SOCIETY \\ CHEVRON RESEARCH CORPORATION \\ NAVAL WEAPONS CENTER
}

The Supporting Institutions listed above contribute to the cost of publication of this Journal, but they are not owners or publishers and have no responsibility for its content or policies.

Mathematical papers intended for publication in the Pacific Journal of Mathematics should be in typed form or offset-reproduced, (not dittoed), double spaced with large margins. Underline Greek letters in red, German in green, and script in blue. The first paragraph or two must be capable of being used separately as a synopsis of the entire paper. The editorial "we" must not be used in the synopsis, and items of the bibliography should not be cited there unless absolutely necessary, in which case they must be identified by author and Journal, rather than by item number. Manuscripts, in duplicate if possible, may be sent to any one of the four editors. Please classify according to the scheme of Math. Rev. Index to Vol. 39. All other communications to the editors should be addressed to the managing editor, Richard Arens, University of California, Los Angeles, California, 90024.

50 reprints are provided free for each article; additional copies may be obtained at cost in multiples of 50 .

The Pacific Journal of Mathematics is published monthly. Effective with Volume 16 the price per volume (3 numbers) is $\$ 8.00$; single issues, $\$ 3.00$. Special price for current issues to individual faculty members of supporting institutions and to individual members of the American Mathematical Society: $\$ 4.00$ per volume; single issues $\$ 1.50$. Back numbers are available.

Subscriptions, orders for back numbers, and changes of address should be sent to Pacific Journal of Mathematics, 103 Highland Boulevard, Berkeley, California, 94708.

PUBLISHED BY PACIFIC JOURNAL OF MATHEMATICS, A NON-PROFIT CORPORATION

Printed at Kokusai Bunken Insatsusha (International Academic Printing Co., Ltd.), 7-17, Fujimi 2-chome, Chiyoda-ku, Tokyo, Japan. 


\section{Pacific Journal of Mathematics}

\section{Vol. 36, No. $3 \quad$ BadMonth, 1971}

E. M. Alfsen and B. Hirsberg, On dominated extensions in linear subspaces of

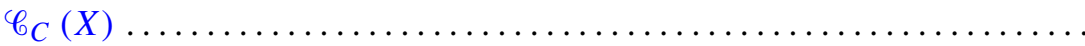

Joby Milo Anthony, Topologies for quotient fields of commutative integral

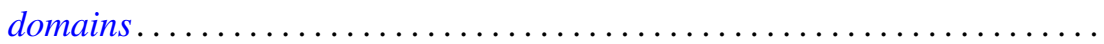

V. Balakrishnan, G. Sankaranarayanan and C. Suyambulingom, Ordered cycle lengths in a random permutation .......................... 603

Victor Allen Belfi, Nontangential homotopy equivalences............... 615

Jane Maxwell Day, Compact semigroups with square roots .............. 623

Norman Henry Eggert, Jr., Quasi regular groups of finite commutative nilpotent algebras .......................................... 631

Paul Erdős and Ernst Gabor Straus, Some number theoretic results ......... 635

George Rudolph Gordh, Jr., Monotone decompositions of irreducible Hausdorff continua .............................................

Darald Joe Hartfiel, The matrix equation $A X B=X \ldots \ldots \ldots \ldots \ldots \ldots . \ldots 69$

James Howard Hedlund, Expansive automorphisms of Banach spaces. II . . . . 671

I. Martin (Irving) Isaacs, The p-parts of character degrees in p-solvable

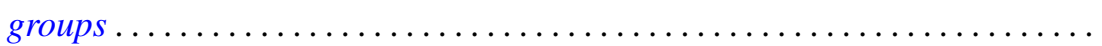

Donald Glen Johnson, Rings of quotients of $\Phi$-algebras ............... 693

Norman Lloyd Johnson, Transition planes constructed from semifield planes....

Anne Bramble Searle Koehler, Quasi-projective and quasi-injective modules.

James J. Kuzmanovich, Completions of Dedekind prime rings as second endomorphism rings...

B. T. Y. Kwee, On generalized translated quasi-Cesàro summability ...

Yves A. Lequain, Differential simplicity and complete integral closure

741

Mordechai Lewin, On nonnegative matrices.

753

Kevin Mor McCrimmon, Speciality of quadratic Jordan algebras ...

Hussain Sayid Nur, Singular perturbations of differential equations in abstract spaces .....................................

D. K. Oates, A non-compact Krein-Milman theorem .

Lavon Barry Page, Operators that commute with a unilateral shift on an invariant subspace...

Helga Schirmer, Properties of fixed point sets on dendrites.

Saharon Shelah, On the number of non-almost isomorphic models of $T$ in a

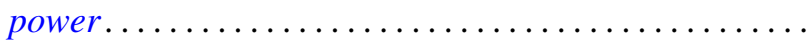

Robert Moffatt Stephenson Jr., Minimal first countable Hausdorff spaces....

Masamichi Takesaki, The quotient algebra of a finite von Neumann algebra 